\title{
Dynamic thiol/disulphide homeostasis in vitiligo patients
}

\author{
Mustafa Aksoyํㅐ, Hakim Çelik ${ }^{2}$ \\ ${ }^{1}$ Department of Skin and Venereal Diseases, Faculty of Medicine, Harran University, Sanlıurfa, Turkey \\ ${ }^{2}$ Department of Physiology, Faculty of Medicine, Harran University, Sanlıurfa, Turkey
}

Adv Dermatol Allergol 2018; XXXV (5): 498-501

DOI: https://doi.org/10.5114/ada.2018.72856

\begin{abstract}
Introduction: Vitiligo is an acquired depigmentation disorder with melanocyte destruction.

Aim: To examine the thiol/disulphide balance in vitiligo patients and to compare the results with a healthy control group.

Material and methods: Thirty-two patients with vitiligo and 35 healthy individuals were included in the study. Native thiol, disulfide and total thiol levels in plasma were evaluated using a new and automated spectrophotometric method. Disulphide/total thiol, disulphide/native thiol and native thiol/total thiol levels were measured.

Results: There was no statistically significant difference between the two groups when the patient and control groups were compared in terms of thiol/disulphide balance $(p>0.05)$. There was no statistically significant difference in native thiol, disulphide and total thiol levels for vitiligo when compared with the control group $(p>0.005)$. Conclusions: In recent years, there have been numerous studies on the role of oxidative stress in the etiopathogenesis of vitiligo. In this study, we investigated in vitiligo patients whether thiol/disulphide balance is a new oxidative stress marker. The results were compared with a healthy control group. We measured the thiol/disulphide balance by a new method developed by Erel and Neselioglu. The serum thiol/disulphide levels were similar in the vitiligo patients and the control subjects, which indicated that the thiol/disulphide balance was not affected by vitiligo. We are of the opinion that new investigations to determine serum levels of thiol/disulphide may shed light on the possible roles of these molecules in vitiligo.
\end{abstract}

Key words: oxidative stress, thiol/disulphide homeostasis, vitiligo.

\section{Introduction}

Vitiligo is a congenital or acquired skin disorder associated with melanocyte destruction and characterised by clinically well-demarcated depigmented macules and patches. Although the definite cause of the ailment has not yet been determined, a number of theories linking it with autoimmune, cytotoxic, biochemical, neural, and oxidant-antioxidant mechanisms have been proposed. The role of viruses and genetics has also been looked into while studying the disease [1, 2]. Vitiligo patients display increased oxidative stress in blood as well as epidermis $[2,3]$.

A variety of biochemical markers are used to define oxidative stress and inflammation. The dynamic thiol/disulphide balance is one of these markers. Thiols, which constitute a significant amount of the total antioxidants in the body, are compounds that contain sulphur and play an important role in helping the body defend against reactive oxygen species. They also play a critically important role in regulating cellular enzymatic activity, antioxidant protection, detoxification, and programmed cell death. The role that plasma thiols play in physiologically cleaning out free radicals and acting as antioxidants with various mechanisms is widely acknowledged [4].

The thiol/disulphide ratio has an important role in detoxification, signal conduction, regulation of enzyme activity, and apoptosis. Deterioration in this ratio can lead to pathogenesis of many inflammatory disorders such as diabetes mellitus, cardiovascular diseases, cancer, rheumatoid arthritis, chronic kidney failure, AIDS, Parkinson's disease, Alzheimer's disease, multiple sclerosis, and liver disorders [4]. Research on the thiol/disulphide balance has determined that it plays a significant role in the pathogenesis of a few skin diseases such as urticaria [5], basal cell carcinoma [6], and psoriasis [7]. Vitiligo is one of the diseases in which oxidative stress is a factor in its etiopathogenesis.

Address for correspondence: Mustafa Aksoy Assist. Prof., Department of Skin and Venereal Diseases, Faculty of Medicine, Harran University, 63200 Sanlıurfa, Turkey, phone: +90 5616110142, e-mail: derma63@gmail.com Received: 8.05.2017, accepted: 10.09.2017. 


\section{Aim}

The aim of this study is to examine the effect of the new oxidative stress indicator, the thiol/disulphide balance in vitiligo patients, and to compare the results with a healthy control group.

\section{Material and methods}

The study was carried out at a dermatology polyclinic of a state university hospital from 1 May 2016 to 1 August 2016. The local Ethics Committee had issued an approval before the study. The study included 32 patients who consulted the polyclinic for their symptoms and were diagnosed with vitiligo for the first time; it also included 35 healthy individuals. Vitiligo was diagnosed by clinical findings and a Wood lamp examination. Vitiligo patients undergoing treatment, pregnant and breastfeeding women, and individuals with any symptom of psychiatric disease were not included in the study. The consent of the participants was taken and a consent form signed by them before the study was carried out.

Venous blood samples were collected from both the patient group and the control group, after at least $8 \mathrm{~h}$ of fasting. These were then centrifuged for $5 \mathrm{~min}$ at $4000 \mathrm{rpm}$ and fractionated. The serums were then stored at $-80^{\circ} \mathrm{C}$ in Eppendorf Tubes.

\section{Measurement of thiol/disulphide homeostasis parameters}

The thiol/disulphide homeostasis tests were measured using a new and fully automatic method developed by Erel and Neselioglu [8]. The disulphide bonds were first reduced to create functional thiol groups together with sodium borohydride. The unused reducing agent, so- dium borohydride, was then removed with formaldehyde in order to prevent the reduction of DTNB (5,5'-dithiobis(2-nitrobenzoic) acid); all thiol groups including the reduced and native thiol groups were determined after the reaction with DTNB. Half of the difference between total thiols and native thiols was determined by the amount of dynamic disulphide. After the native and total thiols were determined, the disulphide levels, disulphide/total thiol, disulphide/native thiol, and native thiol/total thiol ratios were calculated.

\section{Statistical analysis}

The statistical analyses were carried out using IBM SPSS 23.0 (SPSS for Windows, SPSS Inc., Chicago, IL, USA). The Shapiro-Wilk test was used as the normality test. Parametric tests were employed as the distributions were normal. The differences between the groups were examined using the Student $t$-test and Pearson- $\chi^{2}$ test. The limit value for statistically significant differences was 0.05 .

\section{Results}

Of the 32 patients included in the study, 14 were male while 18 were female. The mean age of the patient group was $31.15 \pm 12.27$. Of the 35 healthy individuals included in the study, 19 were male while 16 were female and the mean age of the healthy group was $27.34 \pm 7.16$. Eighteen individuals in the control group were married and 17 were unmarried, 20 individuals in the patient group were married and 12 were unmarried. Of these, 22 individuals in the control group had a regular job while 4 of them had an irregular work schedule, 4 were unemployed and 5 were students; 10 individuals in the patient group had regular jobs while 7 of them had an irregular work sched-

Table 1. Sociodemographic characteristics of the patient and control groups

\begin{tabular}{|c|c|c|c|}
\hline Parameter & Controls $(n=35)$ & Patients $(n=32)$ & $P$-value \\
\hline Age [years] & $27.34 \pm 7.16$ & $31.15 \pm 12.27$ & 0.122 \\
\hline Gender (M/F) & $19 / 16$ & $14 / 18$ & 0.389 \\
\hline Marital status (M/S) & $18 / 17$ & $20 / 12$ & 0.361 \\
\hline Business (RBO/IBO/UE/St) & $22 / 4 / 4 / 5$ & $10 / 7 / 9 / 6$ & 0.065 \\
\hline
\end{tabular}

Table 2. Patient and control group thiol/disulphide levels

\begin{tabular}{lccc}
\hline Parameter & Controls $(n=35)$ & Patients $(n=32)$ & $P$-value \\
\hline Native thiol $(\mathrm{SH})[\mu \mathrm{mol} / \mathrm{l}]$ & $336.3 \pm 63.0$ & $330.8 \pm 56.6$ & 0.710 \\
\hline Total thiol $[\mu \mathrm{mol} / \mathrm{l}]$ & $377.0 \pm 62.0$ & $369.4 \pm 54.4$ & 0.599 \\
\hline Disulphide $(\mathrm{SS})[\mu \mathrm{mol} / \mathrm{l}]$ & $20.3 \pm 5.7$ & $19.3 \pm 6.8$ & 0.500 \\
\hline$\% \mathrm{SS} / \mathrm{SH}$ & $6.31 \pm 2.30$ & $6.08 \pm 2.54$ & 0.690 \\
\hline$\% \mathrm{SS} /$ total thiol & $5.53 \pm 1.78$ & $5.33 \pm 1.98$ & 0.659 \\
\hline$\% \mathrm{SH} /$ total thiol & $88.9 \pm 3.57$ & $89.32 \pm 3.96$ & 0.659 \\
\hline
\end{tabular}


ule, 9 were unemployed and 6 of them were students. There was no statistically significant difference between the patient and control groups in terms of age, sex, marital status, and occupation ( $p>0.005)$. The sociodemographic features and the statistical significance of the patient and control groups are summarised in Table 1.

While the mean thiol/disulphide level in the patient group was $6.08 \pm 2.54$, it was $6.31 \pm 2.30$ in the healthy group. When the difference between the groups was statistically examined, no significant difference was seen between the native thiol, total thiol, and disulphide thiol and the disulphide/native thiol, disulphide/total thiol, and native thiol/total thiol ratios $(p>0.05)$. The relation between the native thiol, total thiol, and disulphide levels and the disulphide/native thiol, disulphide/total thiol, and native thiol/total thiol ratios are summarised in Table 2.

\section{Discussion}

The plasma thiol pool consists fundamentally of protein thiols, albumin thiols, and lower degree low molecular weight thiols. These organic compounds comprise a sulfhydryl group and play a critical role in preventing oxidative stress. The thiol groups of amino acids that contain sulphur such as cysteine and methionine in its protein structure are the primary target of oxygen species. The most common type of thiol found in human cells is the glutathione that creates the optimal redox environment for cell function [8, 9]. Thiol groups create a disulphide bond by oxidation under the impact of reactive oxygen species. The transformation of thiols to disulphides is the earliest indicator of reactive oxygen derivative protein oxidation. Disulfide bond structures can be reduced back to thiol groups. This allows the protection of thiol disulphide homeostasis [10]. It is known that the plasma total thiol level measurement and the determination of thiol/disulphide homeostasis is a good reflection of excess free radical formation in many diseases [4].

Free radicals resulting from normal metabolism or pathological processes cause deterioration in the structure and functions of the thiol bonded enzymes and the thiol/disulphide balance in the cell environment. A decreased plasma thiol concentration shows an increase in free radical generation. Recently, a new method developed by Erel and Neselioglu has made it possible to obtain information about oxidative stress by determining the total plasma thiol/disulphide ratios [8].

Disrupted dynamic thiol/disulphide homeostasis plays a role in various diseases such as myocardial infarction, preeclampsia, polycystic ovary syndrome, diabetes, and cancer [11]. Erel and Neselioglu have shown that the plasma disulphide levels are higher than normal in degenerative diseases such as diabetes, obesity, and pneumonia; in other words, the thiol/disulphide balance leans towards the disulphide. On the other hand, this balance leans towards the thiol in proliferative diseases such as multiple myeloma, bladder cancer, colon cancer, and kidney cancer [8].

The thiol-disulphide balance has been researched in a limited number of skin diseases (urticaria, basal cell carcinoma, psoriasis) until now [5-7]. Demirseren et al. carried out research on 34 patients with basal cell carcinoma and a group of 30 healthy individuals to determine the thiol/disulphide balance. They observed a statistically significant difference between the disulphide/natural thiol and natural thiol/total thiol ratios and the serum native thiol and disulphide levels between the patient and control groups. This established that the thiol/disulphide balance could be a factor in the pathogenesis of basal cell carcinoma [6]. In another study undertaken by Akbas et al., the thiol/disulphide balance was examined in 53 patients with acute urticaria and 47 healthy individuals, and 57 patients with chronic spontaneous urticaria and 57 healthy individuals. While the thiol/disulphide level did not change in the group with acute urticaria, it changed in the group with chronic spontaneous urticaria. This also indicated that there was a decrease in the natural and total thiol levels in both acute urticaria and chronic spontaneous urticaria groups, thus suggesting that the activation of urticaria had an impact on the thiol/disulphide levels [5]. A study carried out by Yazici et al. evaluated the thiol levels in patients with psoriasis and found the serum thiol levels in the patient group to be significantly lower than that of the control group [7].

Numerous studies regarding the examination of the role of oxidative stress in vitiligo patients are available in the existing literature on the subject [12-18]; however, there has been no study as yet that examines the thiol/ disulphide balance in vitiligo patients. Our study researches the new oxidative stress marker and the thiol/ disulphide balance in vitiligo patients and compares the results with a healthy control group. No statistically significant difference was determined between the native thiol, total thiol, and disulphide levels and the disulphide/native thiol, disulphide/total thiol, and the native thiol/total thiol ratios $(p>0.05)$. It was seen that the serum thiol/disulphide levels were similar between vitiligo patients and the control group and that the thiol/ disulphide balance was not affected by vitiligo.

\section{Conclusions}

Even though vitiligo displays high levels of oxidative stress, it is thought that that the oxidative damage is probably not very advanced as there is no sign of disruption of the thiol/disulphide balance between the patient and control groups. Our study needs to be supported by further studies in the future on the levels of thiol derivatives in the blood as well as tissues. 


\section{Conflict of interest}

The authors declare no conflict of interest.

\section{References}

1. Cengiz FP, Emiroglu N, Cevirgen Cemil B, et al. Serum IL-23 levels in patients with vitiligo. Turkderm 2014; 48: 204-7.

2. Yesilova Y, Turan E, Uçmak D, et al. Reduced serum paraoxonase-1 levels in vitiligo: further evidence of oxidative stress. Redox Report 2012; 17: 214-8.

3. Schallreuter KU. Biochemical theory of vitiligo: a role of pteridines in pigmentation. In: Vitiligo. Hann SK, Nordlund JJ (eds). London, Blackwell Science 2000; 151.

4. Ozyazıcı S, Karateke F, Turan U, et al. A novel oxidative stress mediator in acute appendicitis: thiol/disulphide homeostasis. Mediators Inflamm 2016; 2016: 6761050.

5. Akbas A, Sener S, Kılınc F, et al. Investigation of thiol-disulphide balance in patients with acute urticaria and chronic spontaneous urticaria. Cutan Ocul Toxicol 2017; 36: 205-10.

6. Demirseren DD, Cicek C, Alısık M, et al. Dynamic thiol/disulphide homeostasis in patients with basal cell carcinoma. Cutan Ocul Toxicol 2017; 36: 278-82.

7. Yazıcı C, Kose K, Utas S, et al. A novel approach in psoriasis: first usage of known protein oxidation markers to prove oxidative stress. Arch Dermatol Res 2016; 308: 207-12.

8. Erel O, Neselioglu S. A novel and automated assay for thiol/ disulphide homeostasis. Clin Biochem 2014; 47: 326-32.

9. Bal C, Buyuksekerci M, Koca C, et al. The compromise of dynamic disulphide/thiol homeostasis as a biomarker of oxidative stres in trichloroethylene exposure. Hum Exp Toxicol 2016; 35: 915-20.

10. Ates I, Ozkayar N, Inan B, et al. Dinamic thiol/disulphide homeostasis in patients with newly diagnosed primary hypertension. J Am Soc Hypertens 2016; 10: 159-66.

11. Kolgelier S, Ergin M, Demir LS, et al. Impaired thiol-disulphide balance in acute brucellosis. Jpn I Infect Dis 2017; 70: 258-62.

12. Mansur AT, Aydıngoz IE, Goktay F, et al. Serum iron and ferritin levels in patients with vitiligo. Turkderm 2010; 44: 153-5.

13. Yıldırım M, Baysal V, Inaloz HS, et al. The role of oxidants and antioxidants in generalized vitiligo. J Dermatol 2003; 30: 104-8

14. Picardo M, Massi S, Morrone A, et al. Antioxidant status in the blood of patients with active vitiligo. Pigment Cell Res 1994; 7: 110-5.

15. Ines D, Sonia B, Riadh BM, et al. A comparative study of oxidant-antioxidant status in stable and active vitiligo patients. Arch Dermatol Res 2006; 298: 147-52.

16. Kaman D, Demir B. Serum ADMA, MDA, vitamin E and homocysteine levels in vitiligo patients. Firat Med J 2016; 21: 29-34.

17. Zedan H, Abdel-Motaleb AA, Kassem NMA, et al. Low glutathione peroxidase activity levels in patients with vitiligo. J Cutan Med Surg 2015; 19: 144-8.

18. Agrawal S, Kumar A, Dhali TK, et al. Comparison of oxidantantioxidant status in patients with vitiligo and healthy population. Kathmandu Univ Med J 2014; 46: 132-6. 